\title{
The Modified Heinz’s Inequality
}

\author{
Takashi Yoshino \\ Mathematical Institute, Tohoku University, Sendai, Japan \\ Email: yoshino@math.tohoku.ac.jp
}

Received August 23, 2013; revised September 24, 2013; accepted September 28, 2013

Copyright (C) 2013 Takashi Yoshino. This is an open access article distributed under the Creative Commons Attribution License, which permits unrestricted use, distribution, and reproduction in any medium, provided the original work is properly cited.

\section{ABSTRACT}

In my paper [1], we aimed to determine the best possible range of $\gamma$ such that the modified Heinz's inequality $\left(A^{\gamma} A^{\alpha} A^{\gamma}\right)^{\beta} \geq\left(A^{\gamma} B^{\alpha} A^{\gamma}\right)^{\beta}$ holds for any bounded linear operators $A$ and $B$ on a Hilbert space $\mathcal{H}$ such as $A \geq B \geq \tau I$ (some $\tau>0$ ) and for any given $\alpha$ and $\beta$ such as $\alpha>0$ and $\beta>0$. But the counter-examples prepared in [1] and also in [2] were not sufficient and, in this paper, we shall constitute the sufficient counter-examples which will satisfy all the lacking parts.

Keywords: Heinz’s Inequality

By the same way as in the proof of Theorem ([1]), we have the following.

Lemma For any $a, b, x, \delta$ and $\epsilon$ such as $a>1>b>0,0<x<a, 0<\epsilon$ and $0 \leq \delta=O(\epsilon)<a-b$ where $O(\varepsilon)$ is a function of $\epsilon$ such that $\lim _{\epsilon \rightarrow 0} \frac{O(\epsilon)}{\epsilon}$ is bounded, let

$$
A=\left(\begin{array}{cc}
a & \sqrt{\epsilon(a-b-\delta)} \\
\sqrt{\epsilon(a-b-\delta)} & b+\varepsilon+\delta
\end{array}\right) \text { and } B=\left(\begin{array}{cc}
x & 0 \\
0 & b
\end{array}\right) \text {. }
$$

If $\left(A^{\gamma} A^{\alpha} A^{\gamma}\right)^{\beta} \geq\left(A^{\gamma} B^{\alpha} A^{\gamma}\right)^{\beta}$ for any real numbers $\alpha, \beta$ and $\gamma$ such as $\alpha>0$ and $\beta>0$, then we have the following inequality (1).

Theorem ([1]) The region of $\gamma$ such that the operator inequality

$$
\left(A^{\gamma} A^{\alpha} A^{\gamma}\right)^{\beta} \geq\left(A^{\gamma} B^{\alpha} A^{\gamma}\right)^{\beta}
$$

holds for any bounded linear operators $A$ and $B$ on a Hilbert space $\mathcal{H}$ such as $A \geq B \geq \tau I$ (some $\tau>0$ ) and for any given $\alpha$ and $\beta$ such as $\alpha>0$ and $\beta>0$ is as follows:

1) $0<\alpha \leq 1,0<\beta \leq 1,-\infty<\gamma<+\infty$

2) $0<\alpha \leq 1,1<\beta \leq 2$

$\max \left\{-\frac{1}{2}, \frac{-\alpha \beta}{2(\beta-1)}\right\} \leq \gamma \leq \min \left\{0, \frac{1-\alpha \beta}{2(\beta-1)}\right\}$

3) $0<\alpha \leq 1,2<\beta \leq \frac{1}{\alpha}, \gamma=0$

4) $0<\alpha \leq 1,2<\beta$

$\max \left\{\frac{2 \alpha-1-\alpha \beta}{2(\beta-1)}, \frac{-\alpha \beta}{2(\beta-1)}\right\}$

$\leq \gamma \leq \min \left\{\frac{2 \alpha-\alpha \beta}{2(\beta-1)}, \frac{1-\alpha \beta}{2(\beta-1)}\right\}$

and 5) $1<\alpha, 0<\beta<1, \max \left\{0, \frac{\alpha \beta-1}{2(1-\beta)}\right\} \leq \gamma$

$$
\begin{aligned}
& \frac{\beta\left(a^{\alpha \beta}-x^{\alpha \beta}\right) b^{(\alpha+2 \gamma) \beta-2 \gamma}}{\left(x^{\alpha}-b^{\alpha}\right)^{2}}\left\{\frac{\left(a^{2 \gamma}-b^{2 \gamma}\right)\left(x^{\alpha}-b^{\alpha}\right)}{a^{2 \gamma} x^{\alpha}-b^{\alpha+2 \gamma}}-\frac{\alpha(a-b)}{b} \lim _{\epsilon \rightarrow 0} \frac{\delta}{\epsilon}\right\} \\
& \leq \frac{a^{2 \gamma(1-\beta)}}{\left(b^{\alpha+2 \gamma}-a^{2 \gamma} x^{\alpha}\right)^{2}}\left\{a^{(\alpha+2 \gamma) \beta}-b^{(\alpha+2 \gamma) \beta}\right\}\left\{b^{(\alpha+2 \gamma) \beta}-a^{2 \gamma \beta} x^{\alpha \beta}\right\} .
\end{aligned}
$$



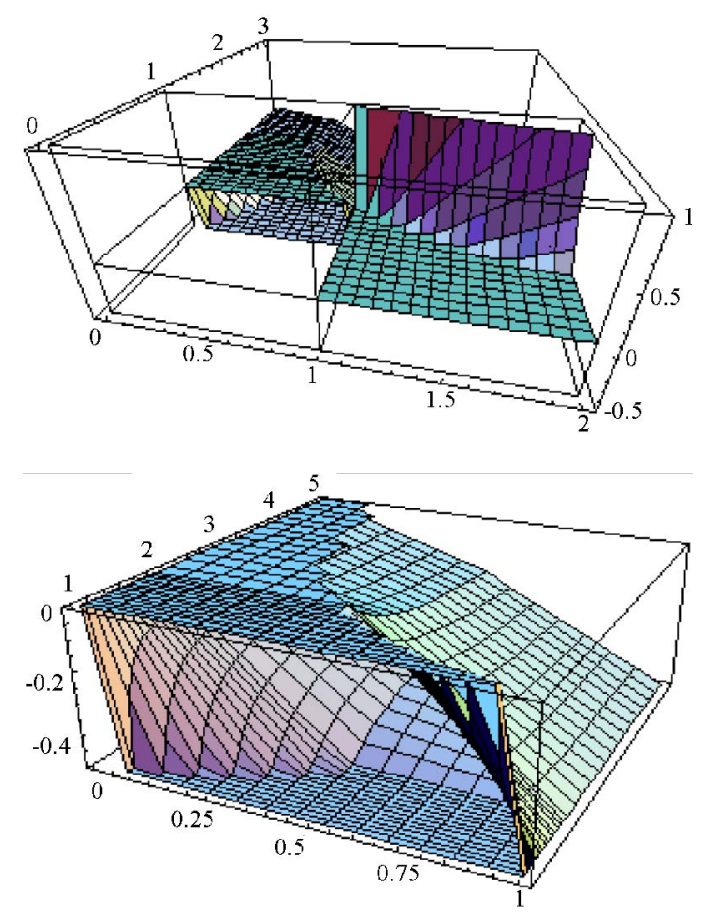

Proof Since the sufficiency of our range for the modified Heinz's inequality is already proved in [1], we have only to constitute counter-examples of $A$ and $B$ in the outside of our ranges.

For any $a, b, x, \delta$ and $\epsilon$ such as in Lemma, let $A=\left(\begin{array}{cc}a & \sqrt{\epsilon(a-b-\delta)} \\ \sqrt{\epsilon(a-b-\delta)} & b+\epsilon+\delta\end{array}\right)$ and $B=\left(\begin{array}{cc}x & 0 \\ 0 & b\end{array}\right)$.

Let $\quad x=b\left\{\frac{(a-1)+a}{(a-1)+b}\right\}=b y, 0<\epsilon<\frac{(a-b)(a-1)}{b}$, and $\delta=\frac{b \epsilon}{a-1}$. Then we have $A \geq B \geq b I$ because $0<\delta<a-b, x=b y>b$,

$$
a-x=\frac{a(a-1)+a b-b(a-1)-b a}{(a-1)+b}=\frac{(a-b)(a-1)}{(a-1)+b}>0
$$

and because

$$
\begin{aligned}
& (a-x)(\epsilon+\delta)-\epsilon(a-b-\delta) \\
& =\epsilon(b-x)+(a-x) \delta+\epsilon \delta \\
& =\frac{\epsilon}{a-1}\{(a-1)(b-x)+(a-x) b\}+\epsilon \delta \\
& =\frac{\epsilon}{a-1}[b\{(a-1)+a\}-x\{(a-1)+b\}]+\epsilon \delta \\
& =\frac{b \epsilon}{a-1}[\{(a-1)+a\}-y\{(a-1)+b\}]+\epsilon \delta=\epsilon \delta>0 .
\end{aligned}
$$

Also if $\left(A^{\gamma} A^{\alpha} A^{\gamma}\right)^{\beta} \geq\left(A^{\gamma} B^{\alpha} A^{\gamma}\right)^{\beta}$, then, by multiply- ing $b^{2 \alpha-\alpha \beta}\left(a^{\alpha \beta}-x^{\alpha \beta}\right)^{-1}$ to the both sides of the inequality (1) in Lemma, we have

$$
\begin{aligned}
& \frac{\beta b^{2 \gamma(\beta-1)}}{\left(y^{\alpha}-1\right)^{2}}\left\{\frac{\left(a^{2 \gamma}-b^{2 \gamma}\right)\left(y^{\alpha}-1\right)}{\left.a^{2 \gamma} y^{\alpha}-b^{2 \gamma}-\frac{\alpha(a-b)}{(a-1)}\right\}}\right. \\
\leq & \frac{a^{2 \gamma(1-\beta)}\left\{a^{(\alpha+2 \gamma) \beta}-b^{(\alpha+2 \gamma) \beta}\right\}\left(b^{2 \gamma \beta}-a^{2 \gamma \beta} y^{\alpha \beta}\right)}{\left(a^{\alpha \beta}-b^{\alpha \beta} y^{\alpha \beta}\right)\left(b^{2 \gamma}-a^{2 \gamma} y^{\alpha}\right)^{2}} \\
= & \frac{\left\{a^{2 \gamma(\beta-1)}-a^{-(\alpha \beta+2 \gamma)} b^{(\alpha+2 \gamma) \beta}\right\}\left(a^{-2 \gamma \beta} b^{2 \gamma \beta}-y^{\alpha \beta}\right)}{\left(1-a^{-\alpha \beta} b^{\alpha \beta} y^{\alpha \beta}\right)\left(a^{-2 \gamma} b^{2 \gamma}-y^{\alpha}\right)^{2}} \\
= & \frac{a^{2 \gamma}\left\{1-a^{-(\alpha+2 \gamma) \beta} b^{(\alpha+2 \gamma) \beta}\right\}\left(b^{2 \gamma \beta}-a^{2 \gamma \beta} y^{\alpha \beta}\right)}{\left(1-a^{-\alpha \beta} b^{\alpha \beta} y^{\alpha \beta}\right)\left(b^{2 \gamma}-a^{2 \gamma} y^{\alpha}\right)^{2}} \\
= & \frac{a^{2 \gamma(1-\beta)-\alpha \beta}\left\{a^{(\alpha+2 \gamma) \beta}-b^{(\alpha+2 \gamma) \beta}\right\}\left(b^{2 \gamma \beta}-a^{2 \gamma \beta} y^{\alpha \beta}\right)}{\left(1-a^{-\alpha \beta} b^{\alpha \beta} y^{\alpha \beta}\right)\left(b^{2 \gamma}-a^{2 \gamma} y^{\alpha}\right)^{2}} .
\end{aligned}
$$

Let $x=1,0<\epsilon<(a-b)(a-1)$ and $\delta=\frac{\epsilon}{a-1}$. Then we have $A \geq B \geq b I$ because $0<\delta<a-b, a>x=1>b$ and because

$$
\begin{aligned}
& (a-x)(\epsilon+\delta)-\epsilon(a-b-\delta)=\epsilon(b-x)+(a-x) \delta+\epsilon \delta \\
& =\epsilon\{(b-1)+1\}+\epsilon \delta=b \epsilon+\epsilon \delta>0 .
\end{aligned}
$$

Also if $\left(A^{\gamma} A^{\alpha} A^{\gamma}\right)^{\beta} \geq\left(A^{\gamma} B^{\alpha} A^{\gamma}\right)^{\beta}$, then, by multiplying $\left(a^{\alpha \beta}-x^{\alpha \beta}\right)^{-1}$ to the both sides of the inequality (1) in Lemma, we have the following inequality

$$
\begin{aligned}
& \frac{\beta b^{(\alpha+2 \gamma) \beta-2 \gamma}}{\left(1-b^{\alpha}\right)^{2}}\left\{\frac{\left(b^{2 \gamma}-a^{2 \gamma}\right)\left(1-b^{\alpha}\right)}{b^{\alpha+2 \gamma}-a^{2 \gamma}}-\frac{\alpha(a-b)}{b(a-1)}\right\} \\
& \leq \frac{a^{2 \gamma(1-\beta)}\left\{a^{(\alpha+2 \gamma) \beta}-b^{(\alpha+2 \gamma) \beta}\right\}\left\{b^{(\alpha+2 \gamma) \beta}-a^{2 \gamma \beta}\right\}}{\left(a^{\alpha \beta}-1\right)\left(b^{\alpha+2 \gamma}-a^{2 \gamma}\right)^{2}} .
\end{aligned}
$$

And, by multiplying $b^{-2(\alpha+2 \gamma)(\beta-1)}$ to the both sides of the above inequality, we have

$$
\begin{aligned}
& \frac{\beta b^{2 \gamma(1-\beta)-\alpha \beta+2 \alpha-1}}{\left(1-b^{\alpha}\right)^{2}}\left\{\frac{b^{1-\alpha}\left(1-b^{-2 \gamma} a^{2 \gamma}\right)\left(1-b^{\alpha}\right)}{1-b^{-(\alpha+2 \gamma)} a^{2 \gamma}}-\frac{\alpha(a-b)}{(a-1)}\right\} \\
& \leq \frac{a^{2 \gamma(1-\beta)}\left\{a^{(\alpha+2 \gamma) \beta} b^{-(\alpha+2 \gamma) \beta}-1\right\}\left\{1-b^{-(\alpha+2 \gamma) \beta} a^{2 \gamma \beta}\right\}}{\left(a^{\alpha \beta}-1\right)\left\{1-b^{-(\alpha+2 \gamma)} a^{2 \gamma}\right\}^{2}} .
\end{aligned}
$$

Let $x=b^{2}$ and $\delta=0$. Then we have $A \geq B \geq b^{2} I$ 
because $0<x=b^{2}<b<a$ and because $(a-x) \epsilon-\epsilon(a-b)=\epsilon(b-x)>0$.

Also if $\left(A^{\gamma} A^{\alpha} A^{\gamma}\right)^{\beta} \geq\left(A^{\gamma} B^{\alpha} A^{\gamma}\right)^{\beta}$, then, by multiplying $b^{-2(\alpha+2 \gamma)(\beta-1)}$ to the both sides of the inequality (1) in Lemma, we have the following inequality

$$
\begin{aligned}
& \frac{\beta\left(a^{\alpha \beta}-b^{2 \alpha \beta}\right) b^{2 \gamma(1-\beta)-\alpha \beta+2 \alpha}}{\left(b^{\alpha}-1\right)^{2}}\left\{\frac{\left(b^{\alpha}-1\right)\left(b^{-2 \gamma} a^{2 \gamma}-1\right)}{b^{\alpha-2 \gamma} a^{2 \gamma}-1}\right\} \\
& \leq \frac{a^{2 \gamma(1-\beta)}\left\{a^{(\alpha+2 \gamma) \beta} b^{-(\alpha+2 \gamma) \beta}-1\right\}\left\{1-b^{(\alpha-2 \gamma) \beta} a^{2 \gamma \beta}\right\}}{\left(1-b^{\alpha-2 \gamma} a^{2 \gamma}\right)^{2}} .
\end{aligned}
$$

For $\beta>2$, let $0<b<\left(\frac{1}{2}\right)^{\beta-1}$. Then $0<b<b^{\frac{1}{\beta-1}}<\frac{1}{2}$ because $0<\frac{1}{\beta-1}<1$.

And let $a>\frac{1-b^{\frac{1}{\beta-1}}-b}{1-2 b^{\frac{1}{\beta-1}}}(>1)$, $x=b^{\frac{\beta}{\beta-1}}\left\{\frac{(a-1)+a}{(a-1)+b}\right\}=b^{\frac{\beta}{\beta-1}} y=b^{\frac{1}{\beta-1}}(b y)(>0)$ and $\delta=0$. Then we have $A \geq B \geq x I$ because

$$
\begin{aligned}
b-x & =\frac{b(a-1)+b^{2}-b^{\frac{\beta}{\beta-1}}(a-1)-b^{\frac{\beta}{\beta-1}} a}{(a-1)+b} \\
& =\frac{b(a-1)\left(1-b^{\frac{1}{\beta-1}}\right)+b\left(b-b^{\frac{1}{\beta-1}} a\right)}{(a-1)+b} \\
& =\frac{b}{(a-1)+b}\left\{(a-1)\left(1-b^{\frac{1}{\beta-1}}\right)+\left(b-b^{\frac{1}{\beta-1}} a\right)\right\} \\
& =\frac{b}{(a-1)+b}\left\{\left(1-2 b^{\frac{1}{\beta-1}}\right) a-\left(1-b^{\frac{1}{\beta-1}}-b\right)\right\}>0,
\end{aligned}
$$

$$
\begin{aligned}
a-x & =\frac{a(a-1)+a b-b^{\frac{\beta}{\beta-1}}(a-1)-b^{\frac{\beta}{\beta-1}} a}{(a-1)+b} \\
& =\frac{\left(a-b^{\frac{\beta}{\beta-1}}\right)(a-1)+a b\left(1-b^{\frac{1}{\beta-1}}\right)}{(a-1)+b}>0
\end{aligned}
$$

and because $(a-x) \epsilon-\epsilon(a-b)=\epsilon(b-x)>0$.

Also if $\left(A^{\gamma} A^{\alpha} A^{\gamma}\right)^{\beta} \geq\left(A^{\gamma} B^{\alpha} A^{\gamma}\right)^{\beta}$, then, by Lemma, we have the following inequality

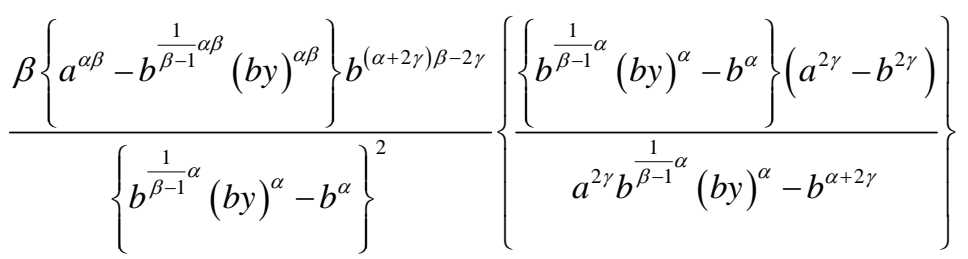

$$
\begin{aligned}
& \leq \frac{a^{2 \gamma(1-\beta)}}{\left\{b^{\alpha+2 \gamma}-a^{2 \gamma} b^{\frac{1}{\beta-1} \alpha}(b y)^{\alpha}\right\}^{2}}\left\{a^{(\alpha+2 \gamma) \beta}-b^{(\alpha+2 \gamma) \beta}\right\}\left\{b^{(\alpha+2 \gamma) \beta}-a^{2 \gamma \beta} b^{\frac{1}{\beta-1} \alpha \beta}(b y)^{\alpha \beta}\right\} \text {. }
\end{aligned}
$$

Since

$$
b^{\frac{1}{\beta-1} \alpha}(b y)^{\alpha}-b^{\alpha}=b^{\frac{1}{\beta-1} \alpha}\left\{(b y)^{\alpha}-b^{\frac{\beta-2}{\beta-1} \alpha}\right\},
$$

$b^{\alpha+2 \gamma}-a^{2 \gamma} b^{\frac{1}{\beta-1} \alpha}(b y)^{\alpha}=b^{\frac{1}{\beta-1} \alpha}\left\{b^{\frac{\beta-2}{\beta-1} \alpha+2 \gamma}-a^{2 \gamma}(b y)^{\alpha}\right\}$,

$$
\begin{aligned}
& b^{(\alpha+2 \gamma) \beta}-a^{2 \gamma \beta} b^{\frac{1}{\beta-1} \alpha \beta}(b y)^{\alpha \beta} \\
& =b^{\frac{1}{\beta-1} \alpha \beta}\left\{b^{\left(\frac{\beta-2}{\beta-1} \alpha+2 \gamma\right) \beta}-a^{2 \gamma \beta}(b y)^{\alpha \beta}\right\}
\end{aligned}
$$

and since $(\alpha+2 \gamma) \beta-\frac{1}{\beta-1} \alpha \beta=\left\{\frac{\beta-2}{\beta-1} \alpha+2 \gamma\right\} \beta$, by multiplying $b^{\frac{\alpha(2-\beta)}{\beta-1}}$ to the both sides of the above inequality, we have, for $\beta>2,0<b<\left(\frac{1}{2}\right)^{\beta-1}$ and $a>\frac{1-b^{\frac{1}{\beta-1}}-b}{1-2 b^{\frac{1}{\beta-1}}}(>1)$, 


$$
\begin{aligned}
& \frac{\beta\left\{a^{\alpha \beta}-b^{\frac{1}{\beta-1} \alpha \beta}(b y)^{\alpha \beta}\right\} b^{\left(\frac{\beta-2}{\beta-1} \alpha+2 \gamma\right) \beta}}{\left\{(b y)^{\alpha}-b^{\frac{\beta-2}{\beta-1}}\right\}^{2}} \\
& \times\left\{\frac{\left.(b y)^{\alpha}-b^{\frac{\beta-2}{\beta-1} \alpha}\right\}\left(a^{2 \gamma} b^{-2 \gamma}-1\right)}{\left.a^{2 \gamma}(b y)^{\alpha}-b^{\frac{\beta-2}{\beta-1} \alpha+2 \gamma}\right\}}\right. \\
& \leq \frac{a^{2 \gamma(1-\beta)}}{\left\{b^{\frac{\beta-2}{\beta-1} \alpha+2 \gamma}-a^{2 \gamma}(b y)^{\alpha}\right\}^{2}} \\
& \times\left\{a^{(\alpha+2 \gamma) \beta}-b^{(\alpha+2 \gamma) \beta}\right\} \\
& \times\left\{b^{\left(\frac{\beta-2}{\beta-1} \alpha+2 \gamma\right) \beta}-a^{2 \gamma \beta}(b y)^{\alpha \beta}\right\} .
\end{aligned}
$$

Case 1 Let $0<\alpha, 1<\beta, 0<\gamma$.

Then

$$
\begin{aligned}
& \lim _{a \rightarrow \infty} \frac{\beta b^{2 \gamma(\beta-1)}}{\left(y^{\alpha}-1\right)^{2}}\left\{\frac{\left(a^{2 \gamma}-b^{2 \gamma}\right)\left(y^{\alpha}-1\right)}{a^{2 \gamma} y^{\alpha}-b^{2 \gamma}}-\frac{\alpha(a-b)}{(a-1)}\right\} \\
& =\frac{\beta b^{2 \gamma(\beta-1)}}{\left(2^{\alpha}-1\right)^{2}}\left\{\frac{2^{\alpha}-1}{2^{\alpha}}-\alpha\right\}
\end{aligned}
$$

because $\lim _{a \rightarrow \infty} y=\lim _{a \rightarrow \infty} \frac{(a-1)+a}{(a-1)+b}=2$ and

$$
\lim _{a \rightarrow \infty} \frac{\left\{a^{2 \gamma(\beta-1)}-a^{-(\alpha \beta+2 \gamma)} b^{(\alpha+2 \gamma) \beta}\right\}\left(a^{-2 \gamma \beta} b^{2 \gamma \beta}-y^{\alpha \beta}\right)}{\left(1-a^{-\alpha \beta} b^{\alpha \beta} y^{\alpha \beta}\right)\left(a^{-2 \gamma} b^{2 \gamma}-y^{\alpha}\right)^{2}}=-\infty .
$$

This contradicts (3).

Case 2 Let $1<\alpha, 0<\beta, \gamma<0$.

Then

$$
\begin{aligned}
& \lim _{a \rightarrow \infty} \frac{\beta b^{2 \gamma(\beta-1)}}{\left(y^{\alpha}-1\right)^{2}}\left\{\frac{\left(a^{2 \gamma}-b^{2 \gamma}\right)\left(y^{\alpha}-1\right)}{a^{2 \gamma} y^{\alpha}-b^{2 \gamma}}-\frac{\alpha(a-b)}{(a-1)}\right\} \\
& =\frac{\beta b^{2 \gamma(\beta-1)}}{\left(2^{\alpha}-1\right)^{2}}\left(2^{\alpha}-1-\alpha\right)
\end{aligned}
$$

because $\lim _{a \rightarrow \infty} y=2$.

If $\alpha+2 \gamma \geq 0$, then we have

$$
\lim _{a \rightarrow \infty} \frac{a^{2 \gamma}\left\{1-a^{-(\alpha+2 \gamma) \beta} b^{(\alpha+2 \gamma) \beta}\right\}\left(b^{2 \gamma \beta}-a^{2 \gamma \beta} y^{\alpha \beta}\right)}{\left(1-a^{-\alpha \beta} b^{\alpha \beta} y^{\alpha \beta}\right)\left(b^{2 \gamma}-a^{2 \gamma} y^{\alpha}\right)^{2}}=0
$$

and, if $\alpha+2 \gamma<0$, then we have also

$$
\begin{aligned}
& \lim _{a \rightarrow \infty} \frac{a^{2 \gamma(1-\beta)-\alpha \beta}\left\{a^{(\alpha+2 \gamma) \beta}-b^{(\alpha+2 \gamma) \beta}\right\}\left(b^{2 \gamma \beta}-a^{2 \gamma \beta} y^{\alpha \beta}\right)}{\left(1-a^{-\alpha \beta} b^{\alpha \beta} y^{\alpha \beta}\right)\left(b^{2 \gamma}-a^{2 \gamma} y^{\alpha}\right)^{2}} \\
& = \begin{cases}0, & (2 \gamma(1-\beta)-\alpha \beta<0) \\
-b^{-\alpha \beta}, & (2 \gamma(1-\beta)-\alpha \beta=0) \leq 0 \\
-\infty, & (2 \gamma(1-\beta)-\alpha \beta>0)\end{cases}
\end{aligned}
$$

and hence, by (4) and (5), we have $2^{\alpha}-1-\alpha \leq 0$ and this contradicts the fact that $2^{\alpha}-1-\alpha>0$ for all $\alpha>1$.

Case 3 Let $0<\alpha, \frac{1}{\alpha}<\beta, \gamma=0$.

Then

$$
\begin{aligned}
& \lim _{a \rightarrow \infty} \frac{\beta b^{2 \gamma(\beta-1)}}{\left(y^{\alpha}-1\right)^{2}}\left\{\frac{\left(a^{2 \gamma}-b^{2 \gamma}\right)\left(y^{\alpha}-1\right)}{a^{2 \gamma} y^{\alpha}-b^{2 \gamma}}-\frac{\alpha(a-b)}{(a-1)}\right\} \\
& =\frac{-\alpha \beta}{\left(2^{\alpha}-1\right)^{2}}
\end{aligned}
$$

and

$$
\begin{aligned}
& \lim _{a \rightarrow \infty} \frac{\left\{a^{2 \gamma(\beta-1)}-a^{-(\alpha \beta+2 \gamma)} b^{(\alpha+2 \gamma) \beta}\right\}\left(a^{-2 \gamma \beta} b^{2 \gamma \beta}-y^{\alpha \beta}\right)}{\left(1-a^{-\alpha \beta} b^{\alpha \beta} y^{\alpha \beta}\right)\left(a^{-2 \gamma} b^{2 \gamma}-y^{\alpha}\right)^{2}} \\
& =\frac{1-2^{\alpha \beta}}{\left(1-2^{\alpha}\right)^{2}}
\end{aligned}
$$

because $\lim y=2$.

By (3), $\stackrel{a \rightarrow \infty}{\text { we have }} 2^{\alpha \beta}-1-\alpha \beta \leq 0$ and this contradicts the fact that $2^{\alpha \beta}-1-\alpha \beta>0$ for all $\alpha \beta>1$.

Case 4 Let $1<\alpha, 0<\beta<1,0<\gamma<\max \left\{0, \frac{\alpha \beta-1}{2(1-\beta)}\right\}$

Then $0<\gamma<\frac{\alpha \beta-1}{2(1-\beta)}$ and $2 \gamma(1-\beta)<\alpha \beta-1$ and hence $(\alpha+2 \gamma) \beta-2 \gamma-1>0$.

Therefore we have

$$
\lim _{b \rightarrow 0} \frac{\beta b^{(\alpha+2 \gamma) \beta-2 \gamma-1}}{\left(1-b^{\alpha}\right)^{2}}\left\{\frac{b\left(b^{2 \gamma}-a^{2 \gamma}\right)\left(1-b^{\alpha}\right)}{b^{\alpha+2 \gamma}-a^{2 \gamma}}-\frac{\alpha(a-b)}{a-1}\right\}=0
$$

and

$$
\begin{aligned}
& \lim _{b \rightarrow 0} \frac{a^{2 \gamma(1-\beta)}\left\{a^{(\alpha+2 \gamma) \beta}-b^{(\alpha+2 \gamma) \beta}\right\}\left\{b^{(\alpha+2 \gamma) \beta}-a^{2 \gamma \beta}\right\}}{\left(a^{\alpha \beta}-1\right)\left(b^{\alpha+2 \gamma}-a^{2 \gamma}\right)^{2}} \\
& =-\frac{a^{(\alpha+2 \gamma) \beta-2 \gamma}}{a^{\alpha \beta}-1}<0 .
\end{aligned}
$$

This contradicts (6). 
Case 5 Let $0<\alpha \leq 1,1<\beta$,

$\gamma<\max \left\{\frac{2 \alpha-1-\alpha \beta}{2(\beta-1)}, \frac{-\alpha \beta}{2(\beta-1)}\right\}$

In this case

$$
\left\{\begin{array}{l}
2 \gamma(\beta-1)+\alpha \beta-\alpha-\max \{\alpha-1,-\alpha\}<0 \\
\text { and }(\alpha+2 \gamma)(\beta-1)<\max \{\alpha-1,-\alpha\} \leq 0 \\
\text { and hence } \alpha+2 \gamma<0 \quad \text { because } \beta>1
\end{array}\right.
$$

Then, in the case where $0<\alpha<\frac{1}{2}$, we have $\gamma<\frac{-\alpha \beta}{2(\beta-1)}$ and hence $2 \gamma(1-\beta)-\alpha \beta>0$. Therefore we have

$$
\begin{aligned}
& \lim _{a \rightarrow \infty} \frac{\beta b^{(\alpha+2 \gamma) \beta-2 \gamma}}{\left(1-b^{\alpha}\right)^{2}}\left\{\frac{\left(b^{2 \gamma}-a^{2 \gamma}\right)\left(1-b^{\alpha}\right)}{b^{\alpha+2 \gamma}-a^{2 \gamma}}-\frac{\alpha(a-b)}{b(a-1)}\right\} \\
& =\frac{\beta b^{(\alpha+2 \gamma) \beta-2 \gamma}}{\left(1-b^{\alpha}\right)^{2}}\left\{\frac{1-b^{\alpha}}{b^{\alpha}}-\frac{\alpha}{b}\right\}
\end{aligned}
$$

and

$$
\begin{aligned}
& \lim _{a \rightarrow \infty} \frac{a^{2 \gamma(1-\beta)-\alpha \beta}\left\{a^{(\alpha+2 \gamma) \beta}-b^{(\alpha+2 \gamma) \beta}\right\}\left\{b^{(\alpha+2 \gamma) \beta}-a^{2 \gamma \beta}\right\}}{\left(1-a^{-\alpha \beta}\right)\left(b^{\alpha+2 \gamma}-a^{2 \gamma}\right)^{2}} \\
& =-\infty .
\end{aligned}
$$

This contradicts (6).

And, in the case where $\frac{1}{2} \leq \alpha \leq 1$, we have $\gamma<\frac{2 \alpha-1-\alpha \beta}{2(\beta-1)}$ and hence $2 \gamma(1-\beta)-\alpha \beta+2 \alpha-1>0$.

Therefore we have

$$
\begin{aligned}
& \lim _{b \rightarrow 0} \frac{\beta b^{2 \gamma(1-\beta)-\alpha \beta+2 \alpha-1}}{\left(1-b^{\alpha}\right)^{2}} \\
& \times\left\{\frac{b^{1-\alpha}\left(1-b^{-2 \gamma} a^{2 \gamma}\right)\left(1-b^{\alpha}\right)}{1-b^{-(\alpha+2 \gamma)} a^{2 \gamma}}-\frac{\alpha(a-b)}{(a-1)}\right\}=0
\end{aligned}
$$

and

$$
\begin{aligned}
& \lim _{b \rightarrow 0} \frac{a^{2 \gamma(1-\beta)}\left\{a^{(\alpha+2 \gamma) \beta} b^{-(\alpha+2 \gamma) \beta}-1\right\}\left\{1-b^{-(\alpha+2 \gamma) \beta} a^{2 \gamma \beta}\right\}}{\left(a^{\alpha \beta}-1\right)\left\{1-b^{-(\alpha+2 \gamma)} a^{2 \gamma}\right\}^{2}} \\
& =-\frac{a^{2 \gamma(1-\beta)}}{a^{\alpha \beta}-1}<0 .
\end{aligned}
$$

This contradicts (7).

Case 6 Let $0<\alpha \leq 1,1<\beta \leq 2, \gamma<-\frac{1}{2}$.

Then $\alpha+2 \gamma \leq 1+2 \gamma<0$ and
$2 \gamma(1-\beta)-\alpha \beta+2 \alpha=2 \gamma(1-\beta)+\alpha(2-\beta)>0$.

And, by (8), we have

$$
\begin{aligned}
0= & \lim _{b \rightarrow 0} \frac{\beta\left(a^{\alpha \beta}-b^{2 \alpha \beta}\right) b^{2 \gamma(1-\beta)-\alpha \beta+2 \alpha}}{\left(b^{\alpha}-1\right)^{2}} \\
& \times\left\{\frac{\left(b^{\alpha}-1\right)\left(a^{2 \gamma} b^{-2 \gamma}-1\right)}{a^{2 \gamma} b^{\alpha-2 \gamma}-1}\right\} \\
\leq & \lim _{b \rightarrow 0} \frac{a^{2 \gamma(1-\beta)}}{\left(1-a^{2 \gamma} b^{\alpha-2 \gamma}\right)^{2}}\left\{a^{(\alpha+2 \gamma) \beta} b^{-(\alpha+2 \gamma) \beta}-1\right\} \\
& \times\left\{1-a^{2 \gamma \beta} b^{(\alpha-2 \gamma) \beta}\right\} \\
= & -a^{2 \gamma(1-\beta)}<0
\end{aligned}
$$

and this is a contradiction.

Case 7 Let $0<\alpha \leq 1,1<\beta, \frac{1-\alpha \beta}{2(\beta-1)}<\gamma<0$.

Then $(\alpha+2 \gamma) \beta>1+2 \gamma>1+\frac{1-\alpha \beta}{\beta-1}=\frac{\beta(1-\alpha)}{\beta-1} \geq 0$ and $\alpha+2 \gamma>0$ because $\beta>1$.

Therefore we have

$$
\lim _{b \rightarrow 0} \frac{\beta b^{(\alpha+2 \gamma) \beta-2 \gamma-1}}{\left(1-b^{\alpha}\right)^{2}}\left\{\frac{b\left(b^{2 \gamma}-a^{2 \gamma}\right)\left(1-b^{\alpha}\right)}{b^{\alpha+2 \gamma}-a^{2 \gamma}}-\frac{\alpha(a-b)}{a-1}\right\}=0
$$

and

$$
\begin{aligned}
& \lim _{b \rightarrow 0} \frac{a^{2 \gamma(1-\beta)}\left\{a^{(\alpha+2 \gamma) \beta}-b^{(\alpha+2 \gamma) \beta}\right\}\left\{b^{(\alpha+2 \gamma) \beta}-a^{2 \gamma \beta}\right\}}{\left(a^{\alpha \beta}-1\right)\left(b^{\alpha+2 \gamma}-a^{2 \gamma}\right)^{2}} \\
& =-\frac{a^{(\alpha+2 \gamma) \beta-2 \gamma}}{a^{\alpha \beta}-1}<0 .
\end{aligned}
$$

This contradicts (6).

Case 8 Let $0<\alpha \leq 1,2<\beta, \frac{2 \alpha-\alpha \beta}{2(\beta-1)}<\gamma<0$.

Then $\alpha+2 \gamma>\frac{\beta-2}{\beta-1} \alpha+2 \gamma>0$.

Since $\lim _{a \rightarrow 1}(b y)=\lim _{a \rightarrow 1} b\left\{\frac{(a-1)+a}{(a-1)+b}\right\}=1$ and since $\lim _{a \rightarrow 1} b=0$ in the case where

$$
\begin{gathered}
\beta>2,0<b<\left(\frac{1}{2}\right)^{\beta-1}, a>\frac{1-b^{\frac{1}{\beta-1}}-b}{1-2 b^{\frac{1}{\beta-1}}}(>1) \text { because } \\
a-1>\frac{b^{\frac{1}{\beta-1}}-b}{1-2 b^{\frac{1}{\beta-1}}}=\frac{b^{\frac{1}{\beta-1}}\left(1-b^{\frac{\beta-2}{\beta-1}}\right)}{1-2 b^{\frac{1}{\beta-1}}}>0,
\end{gathered}
$$


we have

$$
\begin{gathered}
\lim _{a \rightarrow 1} \frac{\beta\left\{a^{\alpha \beta}-b^{\frac{1}{\beta-1} \alpha \beta}(b y)^{\alpha \beta}\right\} b^{\left(\frac{\beta-2}{\beta-1} \alpha+2 \gamma\right) \beta}}{\left\{(b y)^{\alpha}-b^{\frac{\beta-2}{\beta-1} \alpha}\right\}^{2}} \\
\times\left\{\frac{\left\{(b y)^{\alpha}-b^{\frac{\beta-2}{\beta-1}}\right\}\left(a^{2 \gamma} b^{-2 \gamma}-1\right)}{a^{2 \gamma}(b y)^{\alpha}-b^{\frac{\beta-2}{\beta-1} \alpha+2 \gamma}}\right\}=0
\end{gathered}
$$

and

$$
\begin{aligned}
& \lim _{a \rightarrow 1} \frac{a^{2 \gamma(1-\beta)}}{\left\{b^{\frac{\beta-2}{\beta-1} \alpha+2 \gamma}-a^{2 \gamma}(b y)^{\alpha}\right\}^{2}}\left\{a^{(\alpha+2 \gamma) \beta}-b^{(\alpha+2 \gamma) \beta}\right\} \\
& \times\left\{b^{\left(\frac{\beta-2}{\beta-1} \alpha+2 \gamma\right) \beta}-a^{2 \gamma \beta}(b y)^{\alpha \beta}\right\}=-1 .
\end{aligned}
$$

This contradicts (9).

Therefore we completed the proof of the best possibility of the ranges in our theorem.

\section{REFERENCES}

[1] T. Yoshino, “A Modified Heinz's Inequality,” Linear Algebra and its Applications, Vol. 420, No. 2-3, 2007, pp. 686-699. http://dx.doi.org/10.1016/j.laa.2006.08.031

[2] T. Yoshino, "The Best Possible Range of a Modified Heinz's Inequality,” International Journal of Funct. Anal. Oper. Theory Appl., Vol. 3, No. 1, 2011, pp. 1-7. 